Cite this: Soft Matter, 2014, 10, 639

\title{
Pairing of cholesterol with oxidized phospholipid species in lipid bilayers $\dagger$
}

\author{
Himanshu Khandelia, ${ }^{\star a}$ Bastien Loubet, ${ }^{a}$ Agnieszka Olżyńska, ${ }^{b}$ Piotr Jurkiewicz ${ }^{\mathrm{b}}$ \\ and Martin $\mathrm{Hof}^{\mathrm{b}}$
}

We claim that (1) cholesterol protects bilayers from disruption caused by lipid oxidation by sequestering conical shaped oxidized lipid species such as 1-palmitoyl-2-azelaoyl-sn-glycero-3-phosphocholine (PZPC) away from phospholipid, because cholesterol and the oxidized lipid have complementary shapes and (2) mixtures of cholesterol and oxidized lipids can self-assemble into bilayers much like lysolipidcholesterol mixtures. The evidence for bilayer protection comes from molecular dynamics (MD) simulations and dynamic light scattering (DLS) measurements. Unimodal size distributions of extruded vesicles (LUVETs) made up of a mixture of 1,2-dioleoyl-sn-glycero-3-phosphocholine (DOPC) and PZPC containing high amounts of PZPC are only obtained when cholesterol is present in high concentrations. In simulations, bilayers containing high amounts of PZPC become porous, unless cholesterol is also present. The protective effect of cholesterol on oxidized lipids has been observed previously using electron paramagnetic resonance (EPR) and electron microscopy imaging of vesicles. The evidence for the pairing of cholesterol and PZPC comes mainly from correlated 2-D density and thickness plots from simulations, which show that these two molecules co-localize in bilayers. Further evidence that the two molecules can cohabitate comes from self-assembly simulations, where we show that cholesteroloxidized lipid mixtures can form lamellar phases at specific concentrations, reminiscent of lysolipidcholesterol mixtures. The additivity of the packing parameters of cholesterol and PZPC explains their cohabitation in a planar bilayer. Oxidized lipids are ubiquitously present in significant amounts in highand low-density lipoprotein (HDL and LDL) particles, diseased tissues, and in model phospholipid mixtures containing polyunsaturated lipids. Therefore, our hypothesis has important consequences for cellular cholesterol trafficking; diseases related to oxidized lipids, and to biophysical studies of phase behaviour of cholesterol-containing phospholipid mixtures.

Received 2nd September 2013 Accepted 18th November 2013

DOI: $10.1039 / c 3 s m 52310 a$

www.rsc.org/softmatter cholesterol has on bilayers which are assaulted by disrupting agents such as lysolipids. ${ }^{2}$

In 2005, Jacob and Mason ${ }^{1}$ detected the formation of cholesterol domains in bilayers composed of mixtures of polyunsaturated lipids and cholesterol. Specifically, an additional $d$-spacing close to $34 \AA$ was detected, and was interpreted as thinner cholesterol containing regions in the lipid matrix, which also contained thicker, cholesterol-depleted regions enriched in phospholipids. Cholesterol, however, is inverted conical in shape, and like surfactants, cannot form bilayers or bilayer domains on its own, but may do so when coupled with a molecule of complementary shape, such as a lysolipid. ${ }^{3}$ Such a region in a membrane, enriched in cholesterol-lysolipid pairs, will be of a different thickness than the surrounding phospholipid bilayer, and this might explain some of the observations of Jacob and Mason, who observed that the smaller $d$-spacing was only observed in the presence of cholesterol. The natural question that ensues is: where does the lysolipid moiety come from in such an experiment? Peroxidized bilayers do not produce lysolipids, but do produce a large number of oxidized lipid products (OXPLs), the sheer number of which makes it 
impossible to characterize the composition of such a bilayer. PZPC is a typical end-product, OXPL, which is well characterized, found in high concentrations in living and diseased cells, ${ }^{4,5}$ and is implicated in several disease conditions (Fig. 1). PZPC consists of a palmitoyl tail, and a shortened, oxidized acyl tail with a pendant carboxyl group, which reorients to find the lipid-water interface when PZPC is mixed with POPC lipids in a model lipid bilayer $^{6,7}$ (see Fig. 1). In this form, PZPC can be considered to have a very large head group, comprised of the phosphatidylcholine moiety, plus the partially reoriented azealoyl tail. Thus, PZPC acquires a lysolipid like conical shape in a bilayer (Fig. 1). A ternary mixture of phospholipids, cholesterol and PZPC is thus a simple and effective model to test the above hypothesis.

Although we arrived at the above hypothesis independently during our investigations, it is by no means new. LysoPCcholesterol mixtures form bilayers between 45 and $80 \%$ cholesterol (see ref. 3 and references therein). Kumar ${ }^{3}$ noted that although both cholesterol and lysoPCs have antagonistic effects on bilayers at high concentrations, they tend to cancel each other out when both are present in similar proportions. We investigate whether this pairing can also lead to formation of clusters or domains in bilayers. Cholesterol-pairing with lipids containing large head groups is also hypothesized in the so-called "Umbrella Model". ${ }^{8}$

We have implemented coarse-grained Molecular Dynamics (CGMD) simulations of mixtures of DUPC (1,2-dilinoleoyl-snglycero-3-phosphocholine), PZPC and cholesterol to investigate possible lateral reorganization of the lipids resulting in formation of regions rich in both cholesterol and PZPC, and other DUPC-rich regions from which cholesterol and PZPC are excluded. We find that cholesterol and PZPC indeed are colocalized, but this pairing does not lead to explicit domain separation in the simulations. Our finding also explains the mechanism by which cholesterol protects bilayers from peroxidation. ${ }^{9}$ The protective effect of cholesterol has often been ascribed to its ordering effect on bilayers. ${ }^{\mathbf{1 0 - 1 2}}$ We suggest that cholesterol protects bilayers from OXPLs by sequestering them into regions depleted in phospholipids, and are confident that a similar mechanism will also account for cholesterol's protective effects against lysolipids and perhaps other similarly shaped inverted cones.

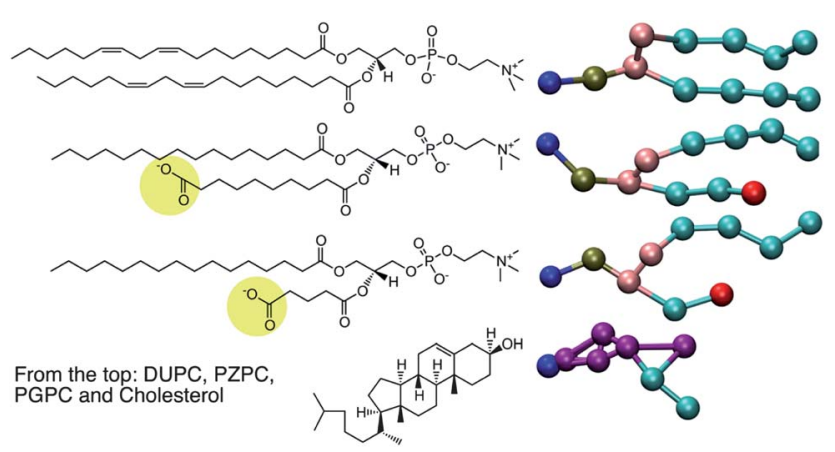

Fig. 1 Left: the chemical structures of the molecules for which MD is carried out. On the right are the coarse-grained representations of the molecules in the MARTINI force field.
The simulations results are complemented by Dynamic Light Scattering (DLS) measurements, which are performed to analyse the morphology of the model lipid membranes, composed of neutral DOPC lipids, oxidized PZPC lipids and cholesterol in various compositions. By measuring the size distribution of extruded liposomes (LUVETs) one can determine whether the size of vesicles is as expected after using this method of preparation (uniform and with the mean size similar to the pores of the membranes used for the extrusion), or if any alterations of vesicle morphology are present. Various types of alterations can be detected. It has been shown that an increased size of LUVETs can be caused by aggregation, which, e.g., might be the effect of the instability of the lamellar phase of lipids. ${ }^{13}$ In the case of LUVETs composed of more than one lipid species, decomposition of the vesicles can occur. This can be often detected using DLS, since the size distribution becomes multimodal in such a case. ${ }^{\mathbf{1 4}}$

Our work has implications for the biomedical and biochemical sciences. OXPLs are known to affect membraneassociated phenomena, and are involved in a variety of disease conditions. ${ }^{5,15-18}$ Oxidized lipids have gained a lot from molecular simulations, ${ }^{19-23}$ because lipid oxidation produces innumerous transient intermediate compounds, which can be tedious to characterize in the lab biochemically and biophysically. Domain separation in models and real membranes is already recognized as a key research area in membrane biophysics, and there have been some reports on lateral reorganization in peroxidized cholesterol-free model membranes. ${ }^{24-27}$ But the problem becomes much richer when cholesterol is also present.

\section{Results and discussion}

\section{Cholesterol protects bilayers from PZPC}

Visual inspection is adequate to determine if each simulated bilayer is intact (able to separate two water compartments) after a few microseconds. Table 1 lists the "final results" from each simulation in the last column.

Without cholesterol, the bilayer first becomes porous at $30 \%$ oxidation. With $30 \%$ cholesterol, the bilayer first becomes porous at $40 \%$ oxidation. With $40 \%$ cholesterol, however, the bilayer is not porous even at $50 \%$ oxidation. Thus, increasing amounts of cholesterol protect the bilayer against increasing amounts of PZPC. A comparison of the final snapshots from the 0-50 (equimolar mixture of DUPC and PZPC) and the 40-50 (equimolar mixture of DUPC and PZPC, and $40 \mathrm{~mol} \%$ cholesterol) is shown in Fig. 2. With the protonated version of PZPC, the bilayer first becomes porous at $40 \%$ oxidation. The protective role of cholesterol is apparent. The data are remarkably consistent with the DLS results.

In DLS, without cholesterol, a unimodal size distribution for each sample is observed up to $40 \%$ of PZPC (Fig. 3A). Note that transient or stable pores, or lateral homogeneity in the bilayer will not be detected by DLS if the vesicle size is unaffected. Beyond 50\% PZPC, the distribution becomes bimodal (Fig. 3A), i.e. a second peak at $\sim 10 \mathrm{~nm}$ is present. We attribute this result to the decomposition of bilayers and formation of small PZPC- 
Table 1 List of completed simulations. Some simulations of the ternary mixtures are implemented with a protonated version of PZPC (see Methods section), and are not shown in this table

Ternary bilayer systems (cholesterol + PZPC + DUPC)

\begin{tabular}{|c|c|c|c|c|}
\hline Name & $\% \mathrm{CHOL}$ & $\%$ oxidation & Onset of bilayer pores & Total simulation time ${ }^{a}(\mu \mathrm{s})$ \\
\hline $0-\mathrm{YY}^{b}$ & 0 & 0 to $70 \%$ at $10 \%$ intervals & At $30 \%$ oxidation & 105 \\
\hline $30-Y Y$ & 30 & 0 to $70 \%$ at $10 \%$ intervals & At $40 \%$ oxidation & 82 \\
\hline & & & & Total $\sim 250 \mu \mathrm{s}$ \\
\hline
\end{tabular}

Binary self-assembly systems (cholesterol + PGPC)

\begin{tabular}{llll}
\hline CHOL $:$ PGPC & Number of simulations & \% simulations in which bilayer forms & Total simulation time $(\mu \mathrm{s})$ \\
\hline $3: 7$ & 3 & 0 & 48 \\
$2: 3$ & 5 & 55.6 & 64 \\
$1: 1$ & 4 & 0 & 52 \\
$3: 2$ & 9 & 0 & 184 \\
$7: 3$ & 3 & 0 & 48 \\
& & & Total $\sim 400 \mu \mathrm{s}$
\end{tabular}

${ }^{a}$ The time shown is actual simulated time multiplied by a factor of 4 , to account for the faster sampling and thermal diffusion rates in coarsegrained simulations. ${ }^{b} \mathrm{YY}$ is the percentage of phospholipids that is oxidized.
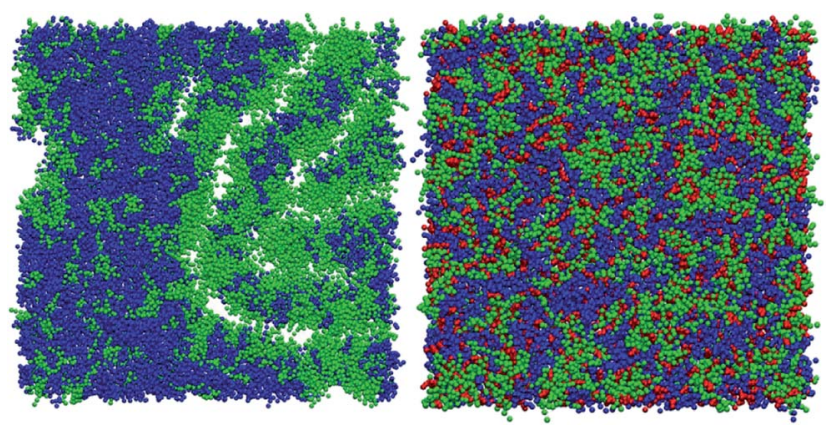

Fig. 2 Final simulation snapshots the bilayer plane of $(A)$ the $0-50$ (DUPC : CHOL : PZPC $0.5: 0: 0.5)$ system and the 40-50 (DUPC : CHOL : PZPC $0.3: 0.4: 0.3)$ system. DUPC in blue, $\mathrm{CHOL}$ in red, and PZPC in green. $40 \%$ cholesterol prevents pore formation in a bilayer containing $50 \%$ oxidized lipids.

rich micelles. This finding supports the results of MD simulations. Samples containing $40 \mathrm{~mol} \%$ cholesterol are characterized by unimodal size distribution up to $40 \mathrm{~mol} \%$ of PZPC (Fig. 3B). The presence of $40 \mathrm{~mol} \%$ cholesterol changes the size distribution of an equimolar DOPC-PZPC from bimodal to unimodal (compare Fig. 3A and B - equimolar samples are underlined). Apparently, cholesterol stabilizes the PZPC-containing bilayer.

\section{Co-localization of PZPC and cholesterol}

PZPC and cholesterol co-localize in the bilayer due to their complementary shapes. In Fig. 4, we show 2-D ensemble averaged density plots of individual molecules in the 40-50 simulation. DUPC is excluded from regions that are enriched in both cholesterol and PZPC. PZPC is clearly concentrated in regions where cholesterol is also present in high concentrations. The PZPC-cholesterol rich clusters are 1 to $4 \mathrm{~nm}$ in size, but do not
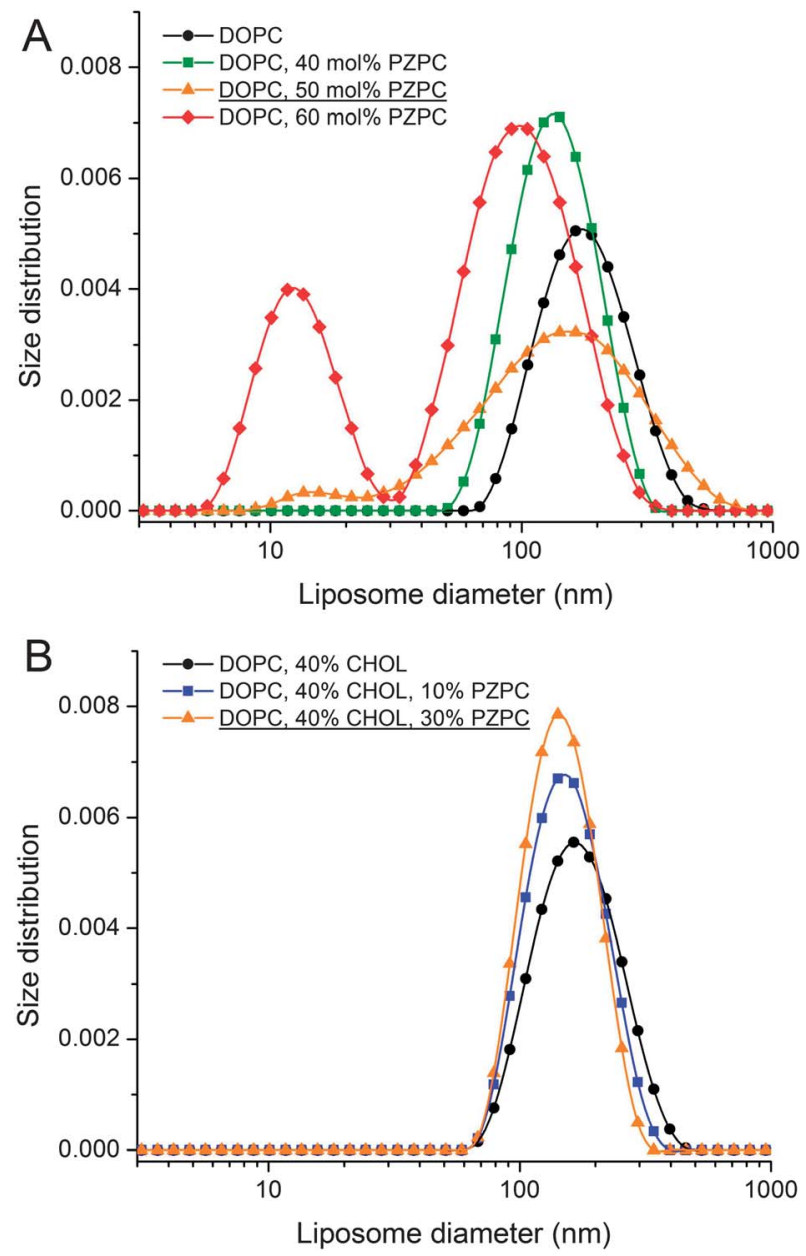

Fig. 3 Dynamic light scattering measurements. (A) Size distribution of extruded liposomes formed from DOPC-PZPC lipids measured at 25 ${ }^{\circ} \mathrm{C}$. (B) Size distribution of extruded liposomes formed from DOPCPZPC and cholesterol measured at $25^{\circ} \mathrm{C}$. Content of the components is given in molar percentages. 

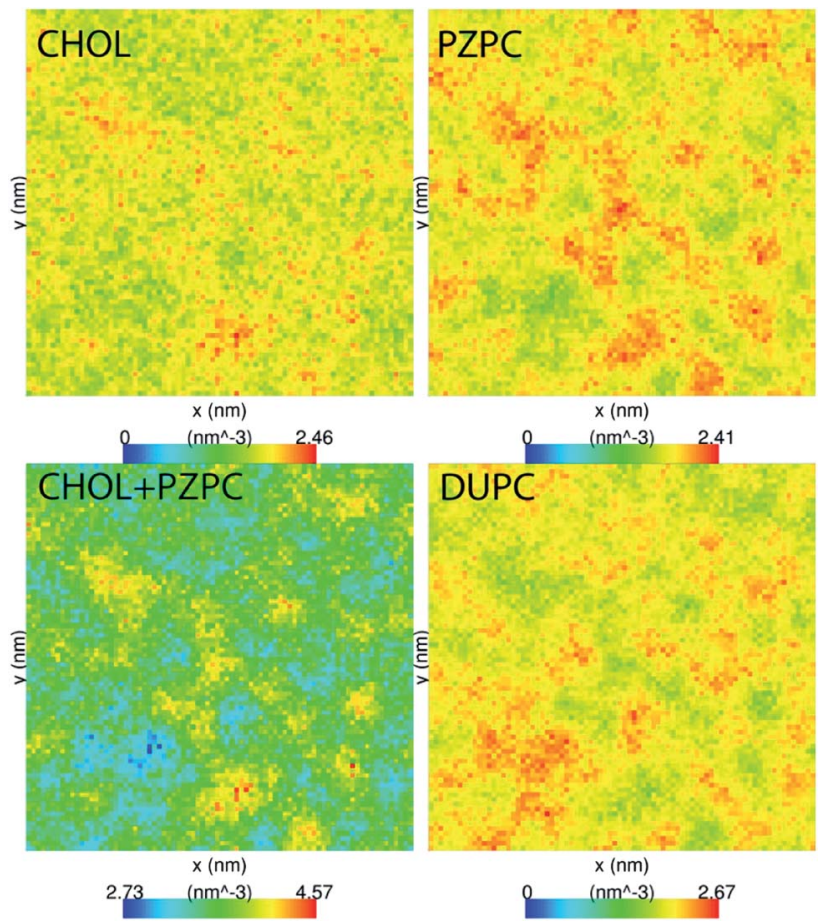

Fig. 4 Average 2D number density of $\mathrm{CHOL}(\mathrm{A}), \mathrm{PZPC}(\mathrm{B}), \mathrm{CHOL}+$ PZPC (C), and DUPC (D) in the 40-50 system. Low-density regions are in blue and the highest densities are in red. $\mathrm{CHOL}$ and PZPC tend to co-localize into nanodomains. The size of the simulation cell is $25 \times$ $25 \mathrm{~nm}$.

acquire a well-defined morphology. Note that cholesterol is not completely excluded from the regions containing DUPC, but is only enriched locally where PZPC is present. Unlike in previous MARTINI-based coarse-grained simulations of archetypal phase-separating systems, ${ }^{28-33}$ we do not observe complete phase separation, but only small transient regions of the bilayer that are enriched in both cholesterol and PZPC. Surprisingly, the cholesterol-PZPC rich regions are thicker than the DUPCrich regions, as evident in the $2 \mathrm{D}$ thickness profiles shown in Fig. 5. For example: the thinnest part of the bilayer at $(x \sim 13, y$ $\sim 3$ ) is rich in DUPC. The minor variation in the thickness (from 3.54 to $3.7 \mathrm{~nm}$ ) suggests that the domains are transient, and complete phase separation has not occurred.

Further evidence of the formation of these "nano-domains" and the increased order in the bilayer comes from measuring the lateral 2-D diffusion coefficient $(D)$ of individual molecules, which decreases nearly monotonically for all components in the mixtures (Fig. 6). Our numbers are of the same order of magnitude as the measured $D$ of labelled OXPLs in a supported DOPC bilayer, ${ }^{34}$ and typical of diffusion coefficients in fluid bilayers. ${ }^{35}$ The decreasing $D$ cannot be ascribed to an ordering of the bilayer induced by cholesterol for the highly oxidized systems, because the order parameters for the lipid tails are in fact lower in the 40-40 and 40-50 systems than in the nonoxidized 40-0 system (data not shown).

In Fig. 7 we show the radial distribution functions of PZPC with DUPC and cholesterol. Although the first and second peaks are identically located, interactions with cholesterol are

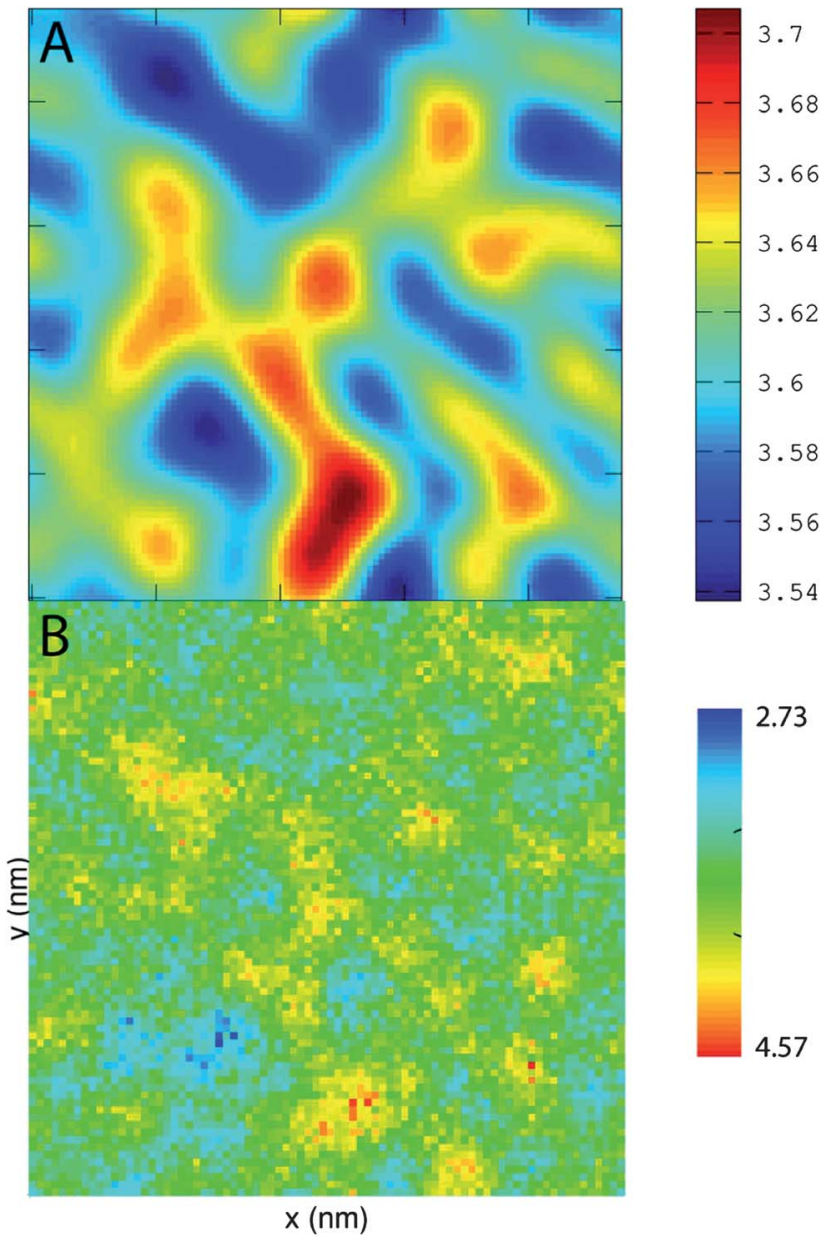

Fig. 5 (A) The thickness of the bilayer in the $x y$-plane in the 40-50 system overlaid with (B) the combined density of $\mathrm{CHOL}+\mathrm{PZPC}$. The axes are $25 \mathrm{~nm} \times 25 \mathrm{~nm}$ on both graphs. Regions rich in cholesterol and PZPC are thicker than the DUPC-rich regions.

stronger, and there is no minimum at $7 \AA$ with cholesterol, suggesting that PZPC prefers to colocalize with cholesterol, than with DUPC.

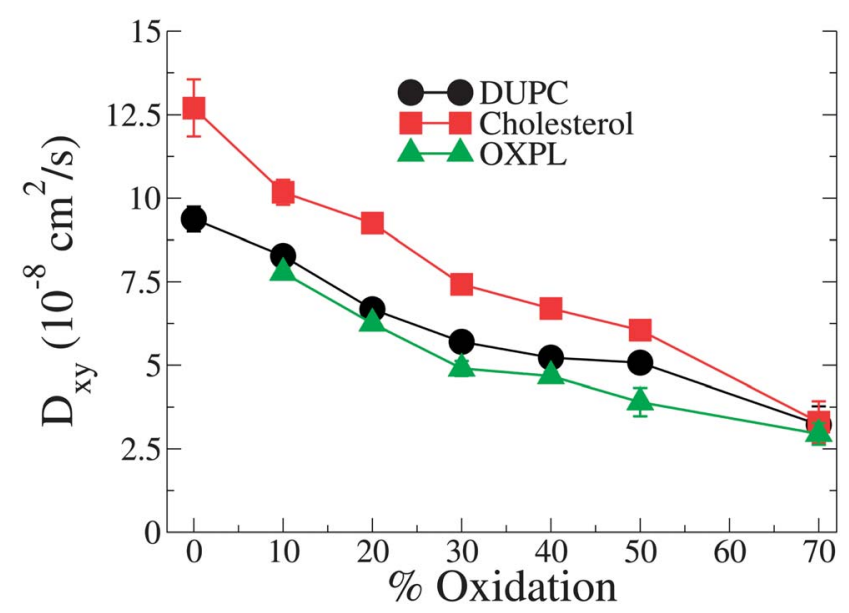

Fig. $6 D$ lateral diffusion constants for cholesterol, PZPC and DUPC as a function of percentage of oxidation for systems containing $40 \%$ cholesterol. 


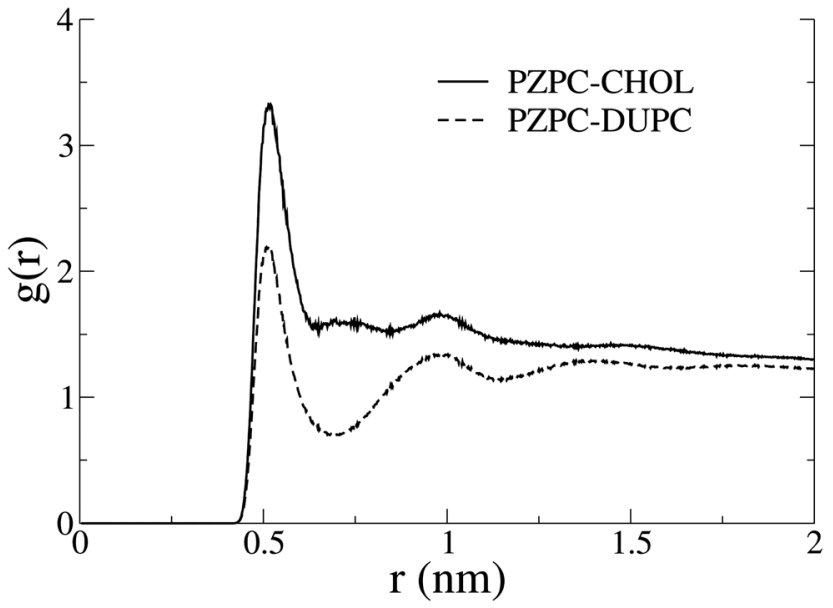

Fig. 7 Radial distribution functions of PZPC with cholesterol and DUPC in the 40-50 system. The higher 1st and 2nd peaks, and the missing minimum between them in the PZPC-cholesterol profile indicate the preference for cholesterol.

If PZPC and cholesterol can co-localize in membranes, it is possible that these two molecules can form planar bilayers much like lysoPC-cholesterol mixtures. ${ }^{3}$ To investigate this possibility, we run self-assembly simulations of mixtures of cholesterol and a PZPC analogue: 1-palmitoyl-2-glutaroyl-snglycero-3-phosphocholine (PGPC), at various cholesterol : PGPC ratios. The PGPC oxidized tail is shorter than PZPC by four methylene groups, corresponding to one MARTINI coarsegrained bead. We select PGPC to run the self-assembly simulations, in order to compare with EPR and electron microscopy experiments carried out on PGPC-cholesterol mixtures. We also run simulations with a protonated version of PGPC, which we call PGHC. PGPC is present in high concentrations in oxidatively modified low-density lipoprotein. ${ }^{36} \mathrm{We}$ find that in 5 out of 9 simulation copies, cholesterol-PGHC mixtures form bilayers at a cholesterol : PGHC ratio of $2: 3$. Bilayers are only formed with the protonated version of PGPC, and only at this specific ratio. Snapshots are shown in Fig. 8. Systems at all other compositions self-assemble into other microstructures such as micelles or bicelles. At CHOL : PGHC $7: 3$ and $3: 2$, micelles are predominant, owing to the surfactant like shape of cholesterol. When the ratio is $1: 1$, bicelles are mostly formed and the bilayer is finally formed when the ratio becomes $2: 3$. The structures formed at $3: 7$ are aggregates of variable, undefined structures. Example simulation snapshots from each of these systems are shown in the ESI, Fig. S1. $\dagger$ The self-assembly simulation result is in wonderful quantitative agreement with Sepharose 4B gel-chromatography and cryo-electron microscopy, ${ }^{9}$ where lamellar structures were only observed at exactly a cholesterol : PGHC ratio of $2: 3$. The self-assembly of PGHCcholesterol mixtures is strong evidence that oxidized phospholipids like PGPC and PZPC behave much like lysoPC. Density profiles from one of the self-assembled simulations are shown in the ESI, Fig. S2. $\dagger$ The carboxylate groups of the oxidized phospholipid and the cholesterol headgroup bead are at identical positions at the bilayer interface, suggesting a possible

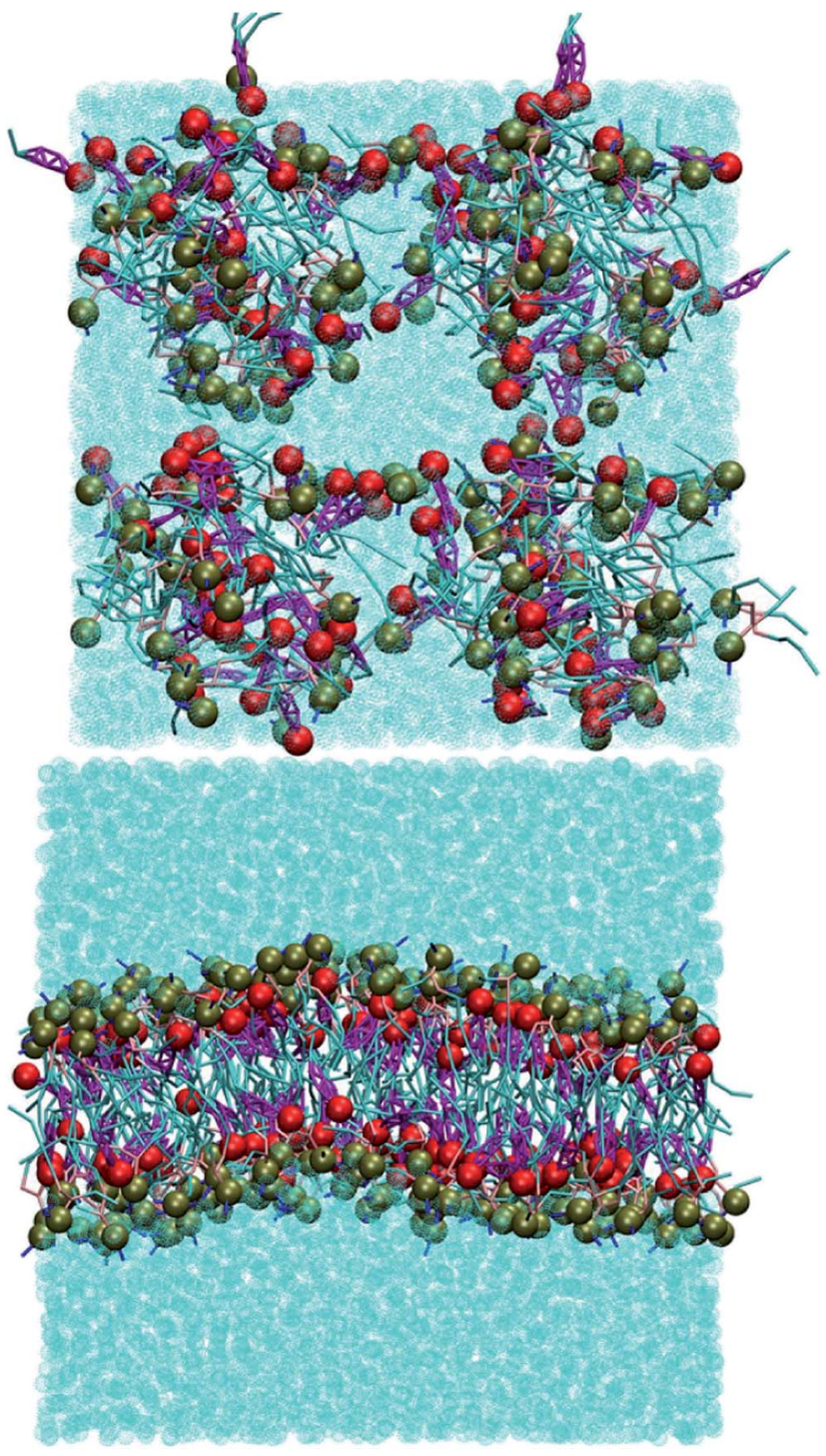

Fig. 8 Starting (A) and final (B) snapshots of a self-assembly simulation of cholesterol: PGPC $=2: 3$. The cholesterol head group is in red. The brown beads represent the phosphate group of PGHC. The bilayer forms in 5 out of 9 simulation copies. In the four other simulations, a bicelle or a porous bilayer is formed. Bilayer assembly only occurs for the protonated version of PGPC.

interaction between these chemical moieties, which however, cannot be further resolved in our CG simulations. The phosphate group of PGPC sits closer to the aqueous phase than the cholesterol headgroup. The non-zero density at the bilayer center for the $\mathrm{COOH}$ group shows that not all the $\mathrm{COOH}$ groups reverse their orientation in the bilayer. The non-zero density of the cholesterol headgroup at the bilayer center is a result of cholesterol flip-flop.

\section{Discussion}

The contention of this report is that (1) cholesterol protects bilayers from disruption caused by lipid oxidation and (2) inverted-conical shaped cholesterol protects the bilayer by 
sequestering conical shaped oxidized lipid species away from the phospholipid, because cholesterol and the oxidized lipid have complementary shapes. We do not decline that the protective effect of cholesterol is also exerted by its ordering of the bilayer, as has been seen with other types of membranedisrupting agents such as drugs and antimicrobial peptides, ${ }^{10-12}$ but argue that this may not be the dominant effect in some cases, such as disruption caused by molecules complementary in shape to cholesterol. For example, the order parameters of the lipid tails are lower in the oxidized bilayers (40-40 and 4050) than in the non-oxidized system, and yet the bilayer is protected. To establish the above assertions, we have implemented about $700 \mu$ s of coarse-grained simulations and carried out simple but informative DLS measurements.

The evidence for bilayer protection comes both from DLS and MD. Unimodal size distributions of extruded vesicles (LUVETs) made of DOPC-PZPC mixtures containing high amounts of PZPC are only obtained when cholesterol is present in high concentrations. Bilayers containing high amounts of PZPC become porous, unless cholesterol is also present. The most striking effect of cholesterol occurs at $50 \%$ oxidation of the bilayer. $40 \%$ cholesterol keeps these membranes intact, as indicated by both the DLS measurements, and the integrity of the bilayer in the MD simulations. The protective effect of cholesterol from oxidized lipids has previously been documented using EPR measurements coupled to images obtained from cyro-electron microscopy. ${ }^{9}$

The evidence for the pairing of cholesterol and PZPC comes from the 2-D density plots from simulations, which show that these two molecules co-localize in bilayers into regions that are thicker than those of the surrounding phospholipid-rich matrix. Furthermore, the lateral diffusion constant of all species in the bilayer mixtures decreases monotonically with increasing extents of oxidation. Further evidence that the two molecules can cohabitate comes from self-assembly simulations, where we show that cholesterol-oxidized lipid mixtures can form lamellar phases at specific concentrations, just like mixtures of cholesterol and lysolipids. ${ }^{3}$ The self-assembly of a cholesterol : PGHC $(2: 3)$ mixture into a bilayer is in very good agreement with cryoelectron microscopy images of vesicle formation by a mixture of identical composition.

The pairing of molecules of conical and inverted conical shapes in bilayers can be understood if terms of the additivity of the packing parameter, ${ }^{3}$ defined as $S=V / a l$, where $V$ is the volume of the hydrocarbon volume, $l$ is the length of the hydrocarbon tail, and $a$ the area of the head group..$^{37,38}$ Lysolipids and PZPC have a large head group (large $V$ ), and $S>1$, and such molecules form micelles. Cholesterol has a much smaller head group, and $S$ is below $1 / 2$. Thus, cholesterol will adopt a hexagonal phase, like phosphatidylethanol lipids. However, when both cholesterol and PZPC are present, they can colocalize, and the additive value of $S$ can be between 0.7 and 1, corresponding to the bilayer-forming regime of $S .^{3}$ The additivity of the packing parameter is sufficient to explain the colocalization of PZPC and cholesterol in our simulations. Our hypothesis on the lateral organization of the ternary lipid mixture is illustrated in Fig. 9.

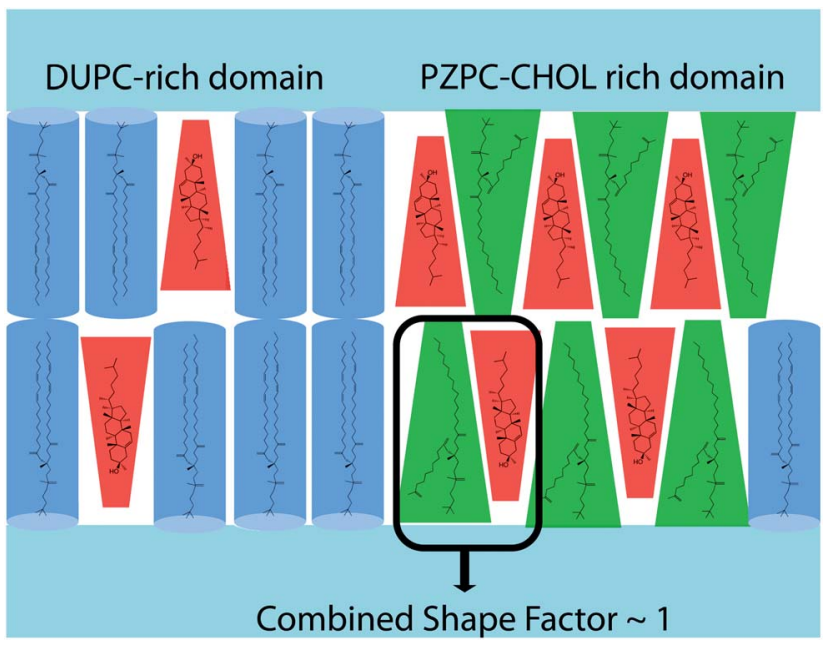

Fig. 9 The hypothesis presented in this report. Cholesterol protects phospholipid bilayers from lipid oxidation products by sequestering oxidation products by shape complementarity. DUPC is shown in blue, PZPC in green and cholesterol in red. PZPC, with a large headgroup prefers to localize with cholesterol, which has a smaller headgroup.

The reduced lateral diffusion coefficient $(D)$ of labelled oxidized lipids in bilayers in the presence of cholesterol was measured in supported lipid bilayers. ${ }^{34}$ Additional MD simulations $^{34}$ ascribed the decreasing $D$ to the deeper location of the oxidized lipid headgroup at higher cholesterol concentrations. Our observations are similar with regard to the decreasing $D$, but we interpret the results differently as described above.

A slightly puzzling issue in the story is that we cannot very clearly detect different phases by means of either radial distribution functions in terms of an outright preference for cholesterol towards one region or another, or dynamically changing the length of the domain interface..$^{28,29,31-33,39-41} \mathrm{We}$ ascribe this anomaly to incomplete phase separation. The largescale heterogeneity in the system masks smaller clusters of PZPC and cholesterol. The radial distribution functions in Fig. 7 do not emulate the compelling nature of the evidence from the 2D density plots and thickness profiles in Fig. 4 and 5. Complete phase separation is possibly driven by other phenomena that would come to the force only if additional degrees of freedom are present in our mixtures, for example: in the form of new molecules, as is probably the case with Jacob and Mason's experiments, ${ }^{1}$ where there are many different phospholipid oxidation products, and possibly cholesterol oxidation products may also be present. We do not think simulation timescales are a problem, because for most canonical phase separating mixtures, phase separation does occur on smaller time scales in MARTINI-based coarse-grained simulations. ${ }^{14,28,29,31-33,40,42}$

Some unresolved questions that still persist are: (1) why does the anionic form of PGPC not form bilayers with cholesterol at all? The uncharged form of PZPC is also less perturbing to the bilayer compared to the anionic form. Electrostatic repulsions between adjoining OXPL species are probably responsible for this effect. (2) What might be the possible role of oxidized cholesterol species in the behavior of these mixtures? (3) Why are PZPC-rich regions slightly thicker than the DUPC-rich 
regions in the ternary simulations? One possibility is that the entire headgroup of PZPC is pulled closer to the aqueous phase because of the chain reversal of the oxidized tail. We are currently addressing some of these questions in additional investigations.

\section{Conclusions}

We present a novel hypothesis of the preferential cohabitation of cholesterol and OXPLs in lipid bilayers, and also propose a new mechanism by which cholesterol may protect bilayers from disrupting agents. Our results are relevant for the health sciences and the membrane biophysics community involved in studying membrane domains in general. PZPC, and similarly shaped PGPC and other OXPLs are present in high concentrations in lipoprotein particles that are also rich in cholesterol..$^{36}$ These OXPLs are also present in apoptotic tissues and are involved in a large variety of ailments, which are reviewed in ref. 43. Mammalian cell membranes contain between 20 and $50 \%$ cholesterol. Therefore the analysis of PZPC-cholesterol interactions is important. Our hypothesis suggests that cholesterol can not only protect mammalian membranes from PZPC, but can also sequester PZPC into cholesterol-PZPC rich regions in the membrane. Such clusters are also likely to form in LDL particles, and will likely affect the overall trafficking of cholesterol in the body. The phase diagrams of cholesterol-containing ternary phase-separating phospholipid mixtures may also need readjustment if there is clear evidence of the presence of oxidized phospholipid species such as PZPC in the mixture.

\section{Experimental}

\section{Simulations}

All simulations are carried out in GROMACS v. 4.5. $\mathrm{x}^{44-47}$ using the MARTINI coarse-grained force field for lipids, ${ }^{48}$ because we anticipate packing to be the main driver of lateral reorganization, and therefore do not attempt to describe specific interactions atomistically. Of course, MARTINI allows simulations of longer time and length scales. A list of the implemented simulations is provided in Table 1. Simulations of bilayers containing various proportions of phospholipid, PZPC and cholesterol are implemented. The model phospholipid used is 1,2-dilinoleoyl-sn-glycero-3-phosphocholine (DUPC), the same as in Jacob and Mason's experiments, but our main conclusions will apply to any typical model phospholipid such as DOPC or POPC. The percentage of oxidation ranged from 0 to $70 \%$ in $10 \%$ intervals, while cholesterol was simulated at 0,30 and $40 \%$ molar percentages. We annotate the simulations as $\mathrm{XX}-\mathrm{YY}$, where $\mathrm{XX}$ is the total percentage of cholesterol present, and YY is the percentage of phospholipids that are oxidized. For example, for a 100-lipid system, 40-30 refers to a system containing 40 molecules of cholesterol, 21 molecules of PZPC and 39 molecules of DUPC. The total number of resulting simulations is 24 . Simulations with 0,30 , and $40 \%$ cholesterol and 20 , 30 and $40 \%$ PZPC are repeated with a protonated version of PZPC, and the results are identical in most respects. We therefore only report results from the deprotonated version for brevity.

We start with a $29 \mathrm{~nm} \times 29 \mathrm{~nm}$ DUPC bilayer patch containing 2250 lipids, 14850 MARTINI water molecules corresponding to 26.4 water molecules per lipid. For systems containing PZPC, we also add an equal number of $\mathrm{Na}^{+}$ions to keep the simulation box electrostatically neutral. Simulations are run for 1 to $5 \mu$ s MARTINI time, corresponding to $4-20 \mu \mathrm{s}$ real simulation time for each simulation. A leapfrog algorithm with a time step of $20 \mathrm{fs}$ was used. Default parameters for nonbonded interactions recommended in the MARTINI force field are used. Simulations are run in the NPT ensemble at $310 \mathrm{~K}$ and $1 \mathrm{~atm}$, with a Berendsen thermo- and barostat. ${ }^{49}$ The coupling time constants are 5 ps and 3 ps for $P$ and $T$ respectively. The aggregate simulation time exceeds $250 \mu \mathrm{s}$.

We also implement self-assembly simulations of mixtures of cholesterol and a PZPC analogue: 1-palmitoyl-2-glutaroyl-snglycero-3-phosphocholine (PGPC), at various cholesterol : PGPC ratios. Although the exact ratios at which PZPC-cholesterol and PGHC (or PGPC)-cholesterol mixtures form bilayers may differ a bit, the fundamental driving force of shape matching is identical. The mixtures contain 300 lipids and 4800 MARTINI water beads. The proportion of cholesterol is varied from 30 to $70 \%$ in $10 \%$ intervals. Simulation parameters are similar to the bilayer simulations. The aggregate simulation time for the selfassembly simulations exceeds $400 \mu \mathrm{s}$.

\section{Dynamic light scattering}

Materials. 1,2-Dioleoyl-sn-glycero-3-phosphocholine (DOPC), 1-palmitoyl-2-azelaoyl-sn-glycero-3-phosphocholine (PZPC) and cholesterol (CHOL) were obtained from Avanti Polar Lipids, Inc. (Alabaster, AL, USA). 4-(2-Hydroxyethyl)-1-piperazineethanesulfonic acid (HEPES) was ordered from Fluka (Buchs, Switzerland). We use DOPC as a model lipid in the DLS measurements to avoid any spontaneous lipid oxidation, which might occur in samples of DUPC. Polyunsaturated lipids get oxidized orders of magnitude faster than monounsaturated ones $^{50}$ and under biological conditions, only the linoleoyl tail is susceptible to oxidation. ${ }^{50,51}$ Furthermore, it was shown long ago that oleic acid protects LDLs from oxidation. ${ }^{52}$ The guarantee that we have no other DUPC oxidation products except PZPC in the samples lends confidence to our interpretation. For the sake of the hypothesis presented in this report, the specific type of phospholipid should not matter as long as the headgroup is identical, and the lipid tails are not vastly different in length or shape. The packing parameter for DOPC was estimated at $1.09 .{ }^{53}$ We could not find the packing parameter for DUPC in the literature. However, it will not be much different from DOPC based on the following arguments. According to the packing theory, the ratio $v / l$ should be unaffected by the increased chain length of DUPC compared to DOPC. ${ }^{54}$ The extra unsaturated bond in DUPC will shorten $l$ by $0.9 \AA^{.54}$ Thus only changes in the area $(a)$ will contribute to differences in the packing parameter, and the difference in the area between DOPC and DUPC is only $7 \% .{ }^{55,56}$ The thickness differs by less than $6 \% .^{57}$ 


\section{Preparation of lipid bilayers}

The required volumes of chloroform solutions of DOPC, PZPC and cholesterol were mixed in a glass tube. Solvents were evaporated under a stream of nitrogen. The lipid film was kept in vacuum for at least 2 hours. The dry lipid film was then suspended in $10 \mathrm{mM}$ HEPES buffer $(\mathrm{pH}=7.4,150 \mathrm{mM} \mathrm{NaCl})$ and vortexed for 5 minutes. The final total concentration of lipids with (or without) cholesterol in the sample was $0.2 \mathrm{mM}$. Extruded large unilamellar vesicles (LUVETs) were formed by extrusion through a polycarbonate membrane (Avestin, Ottawa, Canada) of a nominal pore diameter of $100 \mathrm{~nm}$. All prepared samples were transferred to $1 \mathrm{~cm}$ plastic cuvettes and equilibrated at $25{ }^{\circ} \mathrm{C}$ for 3 minutes before each measurement.

\section{DLS measurements}

Dynamic light scattering (DLS) was used to measure vesicle size distribution. The light scattering setup (Zetasizer Nano ZS, Worcestershire, UK) consisted of a He-Ne laser $532 \mathrm{~nm}$ and an avalanche photodiode detector (APD). The scattering intensity was collected at the angle of $173^{\circ}$. Using cumulant analysis, the mean vesicle size ( $Z$-average) for every monodisperse sample was obtained. The systems analysed are a cholesterol-free DOPC bilayer containing increasing amount of PZPC up to $70 \mathrm{~mol} \%$ and mixtures of $40 \mathrm{~mol} \%$ cholesterol and DOPC with increasing content of PZPC up to $66 \%$.

\section{Acknowledgements}

The computations were done at the SDU node of the Danish Centre for Scientific Computing (DCSC). HK is funded by a Lundbeck Junior Group Leader Investigator Fellowship. AO, PJ, and $\mathrm{MH}$ acknowledge the Grant Agency of the Czech Republic (P106/12/G016). MH acknowledges the Praemium Academie Award. We thank Albin Hermetter and Francesco Megli for useful discussions.

\section{Notes and references}

1 R. F. Jacob and R. P. Mason, J. Biol. Chem., 2005, 280, 3938039387.

2 K. W. Liu and S. L. Biswal, Anal. Chem., 2011, 83, 4794-4801. 3 V. V. Kumar, Proc. Natl. Acad. Sci. U. S. A., 1991, 88, 444-448. 4 S. S. Davies, A. V. Pontsler, G. K. Marathe, K. A. Harrison, R. C. Murphy, J. C. Hinshaw, G. D. Prestwich, A. S. Hilaire, S. M. Prescott, G. A. Zimmerman and T. M. McIntyre, J. Biol. Chem., 2001, 276, 16015-16023.

5 G. O. Fruhwirth, A. Loidl and A. Hermetter, Biochim. Biophys. Acta, Mol. Basis Dis., 2007, 1772, 718-736.

6 H. Khandelia and O. G. Mouritsen, Biophys. J., 2009, 96, 2734-2743.

7 M. E. Greenberg, X. M. Li, B. G. Gugiu, X. D. Gu, J. Qin, R. G. Salomon and S. L. Hazen, J. Biol. Chem., 2008, 283, 2385-2396.

8 J. Huang and G. W. Feigenson, Biophys. J., 1999, 76, $2142-$ 2157.
9 F. M. Megli, E. Conte and T. Ishikawa, Biochim. Biophys. Acta, 2011, 1808, 2267-2274.

10 J. Henriksen, A. C. Rowat, E. Brief, Y. W. Hsueh, J. L. Thewalt, M. J. Zuckermann and J. H. Ipsen, Biophys. J., 2006, 90, 16391649.

11 A. J. McHenry, M. F. M. Sciacca, J. R. Brender and A. Ramamoorthy, Biochim. Biophys. Acta, Biomembr., 2012, 1818, 3019-3024.

12 C. L. Wennberg, D. van der Spoel and J. S. Hub, J. Am. Chem. Soc., 2012, 134, 5351-5361.

13 A. Di Biasio, F. Bordi and C. Cametti, Prog. Colloid Polym. Sci., 2004, 123, 78-82.

14 R. F. Correia, M. I. Viseu, T. J. V. Prazeres and J. M. G. Martinho, J. Colloid Interface Sci., 2012, 379, 56-63.

15 U. Stemmer, C. Ramprecht, E. Zenzmaier, B. Stojcic, G. Rechberger, M. Kollroser and A. Hermetter, Biochim. Biophys. Acta, 2012, 1821, 706-718.

16 A. Hermetter, P. Kinnunen and C. Spickett, Biochim. Biophys. Acta, 2012, 1818, 2373.

17 U. Stemmer, Z. A. Dunai, D. Koller, G. Purstinger, E. Zenzmaier, H. P. Deigner, E. Aflaki, D. Kratky and A. Hermetter, Lipids Health Dis., 2012, 11, 110.

18 T. M. McIntyre, Biochim. Biophys. Acta, 2012, 1818, 24562464.

19 P. Jurkiewicz, A. Olzynska, L. Cwiklik, E. Conte, P. Jungwirth, F. M. Megli and M. Hof, Biochim. Biophys. Acta, 2012, 1818, 2388-2402.

20 L. Beranova, L. Cwiklik, P. Jurkiewicz, M. Hof and P. Jungwirth, Langmuir, 2010, 26, 6140-6144.

21 M. Vazdar, P. Jurkiewicz, M. Hof, P. Jungwirth and L. Cwiklik, J. Phys. Chem. B, 2012, 116, 6411-6415.

22 J. Wong-Ekkabut, Z. Xu, W. Triampo, I. M. Tang, D. P. Tieleman and L. Monticelli, Biophys. J., 2007, 93, 4225-4236.

23 A. Hermetter, W. Kopec and H. Khandelia, Biochim. Biophys. Acta, 2013, 1828, 1700-1706.

24 F. M. Megli, L. Russo and K. Sabatini, FEBS Lett., 2005, 579, 4577-4584.

25 R. P. Mason, M. F. Walter and P. E. Mason, Free Radical Biol. Med., 1997, 23, 419-425.

26 U. Muscatello, A. Alessandrini, G. Valdre, V. Vannini and U. Valdre, Biochem. Biophys. Res. Commun., 2000, 270, 448-452.

27 J. Zhao, J. Wu, H. L. Shao, F. Kong, N. Jain, G. Hunt and G. Feigenson, Biochim. Biophys. Acta, Biomembr., 2007, 1768, 2777-2786.

28 S. Baoukina, E. Mendez-Villuendas, W. F. D. Bennett and D. P. Tieleman, Faraday Discuss., 2013, 161, 63-75.

29 H. J. Risselada and S. J. Marrink, Proc. Natl. Acad. Sci. U. S. A., 2008, 105, 17367-17372.

30 Q. Waheed, R. Tjornhammar and O. Edholm, Biophys. J., 2012, 103, 2125-2133.

31 C. Rosetti and C. Pastorino, J. Phys. Chem. B, 2012, 116, 35253537.

32 D. Hakobyan and A. Heuer, J. Phys. Chem. B, 2013, 117, 38413851.

33 R. S. Davis, P. B. S. Kumar, M. M. Sperotto and M. Laradji, J. Phys. Chem. B, 2013, 117, 4072-4080. 
34 B. Plochberger, T. Stockner, S. Chiantia, M. Brameshuber, J. Weghuber, A. Hermetter, P. Schwille and G. J. Schutz, Langmuir, 2010, 26, 17322-17329.

35 G. Lindblom and G. Oradd, Prog. Nucl. Magn. Reson. Spectrosc., 1994, 26, 483-515.

36 G. O. Fruhwirth, A. Moumtzi, A. Loidl, E. Ingolic and A. Hermetter, Biochim. Biophys. Acta, 2006, 1761, 1060-1069.

37 J. N. Israelachvili and D. J. Mitchell, Biochim. Biophys. Acta, 1975, 389, 13-19.

38 J. N. Israelachvili, D. J. Mitchell and B. W. Ninham, J. Chem. Soc., Faraday Trans. 2, 1976, 72, 1525-1568.

39 P. W. Tumaneng, S. A. Pandit, G. J. Zhao and H. L. Scott, J. Chem. Phys., 2010, 132, 065104.

40 D. V. Pyrkova, N. K. Tarasova, N. A. Krylov, D. E. Nolde and R. G. Efremov, Biol. Membr., 2011, 28, 298-306.

41 P. S. Niemela, S. Ollila, M. T. Hyvonen, M. Karttunen and I. Vattulainen, PLoS Comput. Biol., 2007, 3, e34.

42 J. de Joannis, P. S. Coppock, F. C. Yin, M. Mori, A. Zamorano and J. T. Kindt, J. Am. Chem. Soc., 2011, 133, 3625-3634.

43 P. K. J. Kinnunen, K. Kaarniranta and A. K. Mahalka, Biochim. Biophys. Acta, Biomembr., 2012, 1818, 2446-2455.

44 H. J. C. Berendsen, D. van der Spoel and R. van Drunen, Comput. Phys. Commun., 1995, 91, 43-56.

45 B. Hess, C. Kutzner, D. van der Spoel and E. Lindahl, J. Chem. Theory Comput., 2008, 4, 435-447.
46 E. Lindahl, B. Hess and D. van der Spoel, J. Mol. Model., 2001, 7, 306-317.

47 D. V. D. Spoel, E. Lindahl, B. Hess, G. Groenhof, A. E. Mark and H. J. C. Berendsen, J. Comput. Chem., 2005, 26, 17011718.

48 S. J. Marrink, H. J. Risselada, S. Yefimov, D. P. Tieleman and A. H. de Vries, J. Phys. Chem. B, 2007, 111, 7812-7824.

49 H. J. C. Berendsen, J. P. M. Postma, W. F. Vangunsteren, A. Dinola and J. R. Haak, J. Chem. Phys., 1984, 81, 3684-3690.

50 N. A. Porter, L. S. Lehman, B. A. Weber and K. J. Smith, J. Am. Chem. Soc., 1981, 103, 6447-6455.

51 T. M. McIntyre, G. A. Zimmerman and S. M. Prescott, J. Biol. Chem., 1999, 274, 25189-25192.

52 S. Parthasarathy, J. C. Khoo, E. Miller, J. Barnett, J. L. Witztum and D. Steinberg, Proc. Natl. Acad. Sci. U. S. A., 1990, 87, 3894-3898.

53 D. Marsh, Biophys. J., 1997, 72, 2834-2836.

54 J. N. Israelachvili, D. J. Mitchell and B. W. Ninham, Biochim. Biophys. Acta, 1977, 470, 185-201.

55 N. Kucerka, J. F. Nagle, J. N. Sachs, S. E. Feller, J. Pencer, A. Jackson and J. Katsaras, Biophys. J., 2008, 95, 2356-2367.

56 M. Stepniewski, A. Bunker, M. Pasenkiewicz-Gierula, M. Karttunen and T. Rog, J. Phys. Chem. B, 2010, 114, 11784-11792.

57 W. Rawicz, K. C. Olbrich, T. McIntosh, D. Needham and E. Evans, Biophys. J., 2000, 79, 328-339. 